\title{
On the dispatch of minigrids with large penetration levels of variable renewable energy
}

\author{
Anan A. Dweekat, Mohamed Shaaban, Sze Song Ngu \\ Department of Electrical and Electronic Engineering, Universiti Malaysia Sarawak (UNIMAS), Sarawak, Malaysia
}

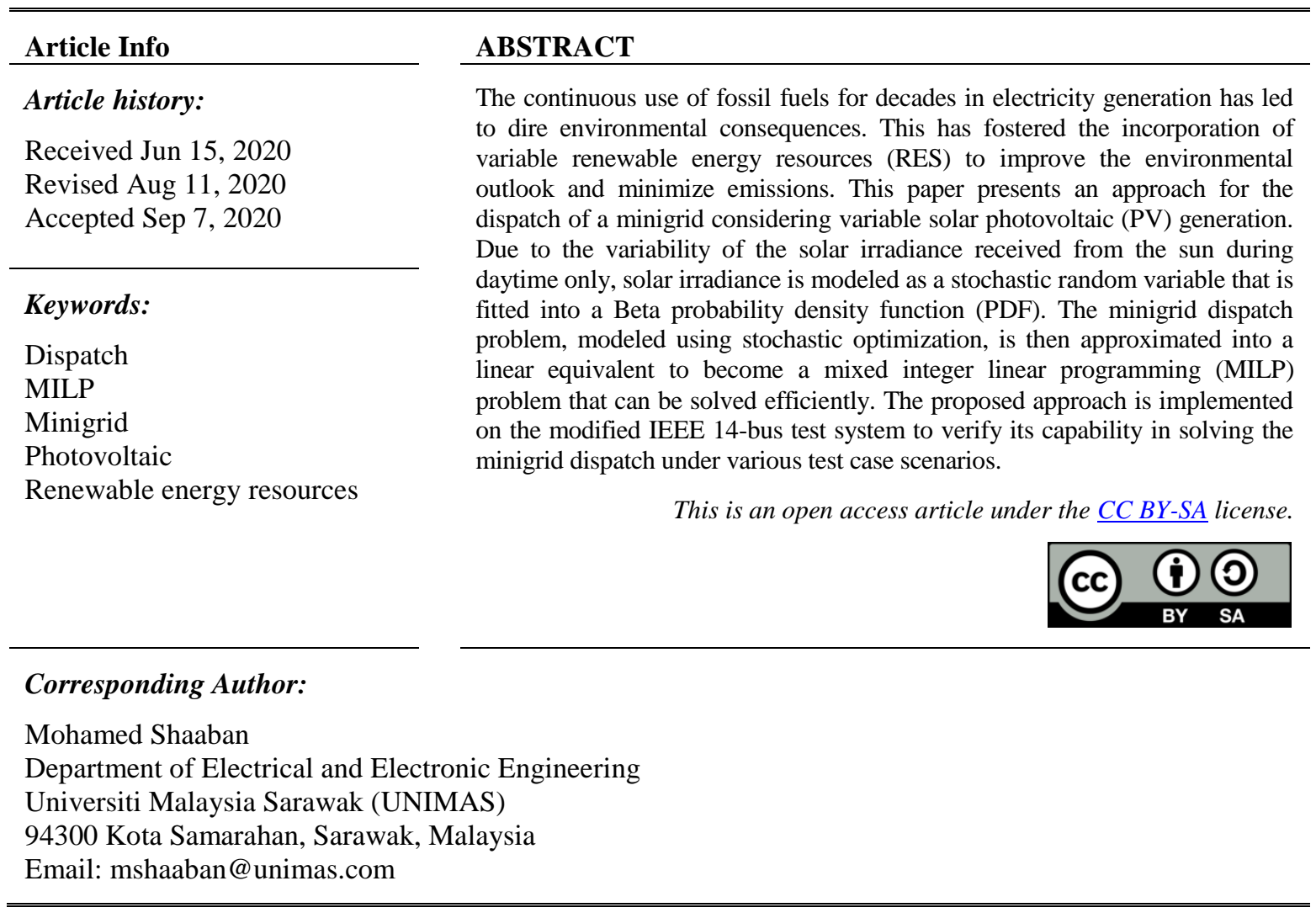

\section{INTRODUCTION}

The negative environmental impacts associated with using fossil fuels in conventional generation have prompted the adoption of renewable energy sources (RES) in large proportions to achieve a more efficient and environment-friendly energy delivery ecosystem [1,2]. RES is integrated to the power grid as distributed generations (DGs) connected at the distribution system level [3-5]. DGs have many advantages such as their capability of reducing environmental emissions and operation costs, as well as avoiding expensive power system expansions, when DGs are used in standalone minigrid systems in rural areas [6]. On the other hand, increasing the penetration level of variable RES in power systems will subsequently increase the uncertainty [7, 8]. This is due to the intermittency nature of variable RES, which are quite sensitive to weather conditions and may not be available at load demand peak hours [9].

Many countries around the world are developing their energy policies to integrate RES, including solar PV, hydropower and biomass, into their energy mix [10]. A minigrid is a local network in a small geographical area that operates disconnected from the main utility grid [11]. The minigrid has DG units as energy sources, which can be small conventional generators and/or RES such as solar PV, and load demand of end users [12]. Solar energy is a variable RES that can be employed in electricity generation to deliver electricity to rural areas [6]. Solar energy is a viable option for energy supply in rural villages, which are more than $30 \mathrm{~km}$ away from the main grid, in the state of Sarawak, Malaysia. A hybrid minigrid, that contains both conventional generation and solar PV panels, provides a more reliable, sustainable, and economical alternative to grid expansion [13]. 
Solar PV is among the category of variable RES. Solar irradiance, which is a measure of how much solar power is received, has a direct relation to the energy generated from solar PV panels [14]. Solar PV energy output thus depends on sunshine hours, clouds, and other related weather conditions [15]. The optimal generation dispatch is therefore a challenging problem to sustain power system reliability, flexibility and satisfy load demand under all deterministic/ non-deterministic conditions [16]. Scheduling of the minigrid determines the hourly operation timetable of the committed DGs, including conventional generating units and RES, to satisfy the hourly load demand with the lowest operation cost over a 24-hour timespan [17]. To consider the uncertainty of RES in power system problems, stochastic optimization techniques are used; such as full stochastic programming, chance-constrained programming and robust optimization [18]. The traditional, and widely used, method to represent the uncertainty is scenario generation in full stochastic programming [19]. However, scenario generation techniques are computationally inefficient [18].

In this paper, a model is proposed to dispatch a minigrid that consists of small conventional generators and a large penetration level of solar PV energy sources, as well as a residential load demand. The solar irradiance of the solar PV is modeled as a random variable using a probability density function (PDF) called Beta distribution to represent its uncertainty in the stochastic optimization approach. The resulting nonlinear stochastic scheduling problem is approximated into a linear equivalent to be solved by the efficient mixed integer linear programming (MILP) technique. The latter, MILP, is widely adopted by the utility industry due to its accuracy in achieving optimal solutions in large-scale problems [20]. The structure of this paper is as follows. Problem formulation is outlined in Section 2. Section 3 describes the numerical simulations in detail. Conclusions are presented in Section 4.

\section{PROBLEM FORMULATION}

Minimizing the total operation cost is the main objective of the proposed minigrid scheduling approach, taking into account the technical and operational constraints of conventional generation as well as solar energy penetration level. The total operation cost required for minigrid dispatch consists of three parts: fuel cost, startup cost, and shutdown cost. Solar energy's operation cost is negligible, and is modeled as a negative load as described in the following.

\subsection{Objective function}

The generic objective function is given:

$$
\min \sum_{i, t}^{N, T} F C_{i}(t)+S T C_{i}(t)+S D C_{i}(t)
$$

where $F C_{i}(t)$ is the fuel cost function of conventional generating units. $S T C_{i}(t)$ is the startup cost function which is exponentially increasing, and $S D C_{i}(t)$ is the shutdown cost function. The nonlinearities associated with the quadratic fuel cost function and the exponential increasing function of the startup cost increase the computational burden of the dispatch problem. Therefore, all nonlinearities are approximated into a linear form and formulated to fit the MILP structure, following the model presented in [21]. The fuel cost equation is represented as:

$$
F C_{i}(t)=a_{i} P_{i}^{2}+b_{i} P_{i}+c_{i}
$$

where $a_{i}, b_{i}$ and $c_{i}$ are the fuel cost constants and $P_{i}$ is the output power of unit $i$. The fuel cost function is quadratic. It is approximated into a linear equivalent as [21]:

$$
F C_{i}(t)=A_{i} u_{i, t}+\sum_{k} S_{i}^{k} P_{i, t}^{k}
$$

where $A_{i}=a_{i} P_{i \text { min }}^{2}+b_{i} P_{i \text { min }}+c_{i}, u_{i, t}$ is the status of the generating unit $i$ at time $t, S_{i}^{k}$ is the slope of the stepwise $k$ and unit $i$ and $P_{i, t}^{k}$ is the output power of unit $i$ at time $t$ and block number $k$.

$$
\begin{aligned}
& 0 \leq P_{i, t}^{k} \leq \Delta P_{i}^{k} \\
& \Delta P_{i n i}^{k} \leq P_{i n i}^{k}-P_{i \min } \\
& P_{i, t}^{k}=\sum_{k} S_{i}^{k} P_{i, t}^{k}+P_{i \min } \\
& \Delta P_{i}^{k}=\frac{P_{\text {imax }}-P_{i \min }}{n}
\end{aligned}
$$


where $P_{i n i}^{k}$ is the output power at the starting point of block $k, P_{i \text { max }} / P_{i \text { min }}$ are the maximum and minimum output power of unit $i$ respectively and $n$ is the number of blocks.

The stairwise approximation of the startup cost function is:

$$
S T C_{i}(t) \geq S C_{k, i}\left[u_{i, t}-\sum_{n=1}^{t}\left(u_{i, t-n}\right)\right]
$$

where $S C_{k, i}$ is unit $i$ startup cost and $u_{i, t}$ is the unit status at time $t$.

The shutdown cost equation is expressed as:

$$
S D C_{i}(t) \geq D C_{i}\left(u_{i, t-1}-u_{i, t}\right)
$$

where $D C_{i}$ is the shutdown cost of the generating unit $i$.

\subsection{Operational and technical constraints}

Conventional generation have technical and operational constraints. These are described as follows: Ramp up/down constraint: the capability of a generating unit to increase its generation, without exceeding a maximum limit of increment, is called the ramp up. Similarly, the action of a unit to decrease its generated output power to a lower value is called ramp down. Mathematically, ramping equations can be expressed as:

$$
\begin{aligned}
& P_{i \max }(t) \leq P_{i}(t-1)+R U_{i} u_{i, t-1}+S U_{i}\left(u_{i, t}-u_{i, t-1}\right)+P_{i \max }\left(1-u_{i, t}\right) \\
& P_{i \max }(t) \leq P_{i \max } u_{i, t+1}+S D_{i}\left(u_{i, t}-u_{i, t+1}\right) \\
& P_{i}(t-1)-P_{i}(t) \leq R D_{i} u_{i, t}+S D_{i}\left(u_{i, t-1}-u_{i, t}\right)+P_{i \max }\left(1-u_{i, t-1}\right)
\end{aligned}
$$

where $P_{i, t}$ is the output power of the generating unit $i$ at time $t, R U_{i}$ and $R D_{i}$ are the ramping up/down of the generating units respectively. $S U_{i}$ and $S D_{i}$ are the startup ramp limit and the shutdown ramp limit of unit $i$ respectively. Minimum up/down time constraint: when a conventional generating unit is turned off after being committed, it has a minimum time to stay off before turning it on again. This time is called the minimum down time. If the unit was off and turned on, it has a minimum on time, called up time, before decommitting it again. The equations are modeled with a single binary variable to enhance the model computational efficiency as:

$$
\begin{aligned}
& \sum_{t=k}^{K+U T_{i-1}} u_{i, t} \geq U T_{i}\left(u_{i, t}-u_{i, t-1}\right) \\
& \sum_{t=k}^{K+D T_{i-1}}\left(1-u_{i, t}\right) \geq D T_{i}\left(u_{i, t-1}-u_{i, t}\right)
\end{aligned}
$$

The uptime of the generating unit is $U T_{i}$ and the downtime is $D T_{i}$. Generation output power limit: generating unit output power is limited by a maximum and minimum capacity limits as follows:

$$
P_{i \min } \leq P_{i, t} \leq P_{i \max }
$$

Transmission line capacity limits: network's transmission lines of the power system have thermal limitations on its capacity for power delivery. The limits can be expressed as:

$$
P_{i j}^{\min } \leq P_{i j} \leq P_{i j}^{\max }
$$

where $P_{i j}$ is the power delivered between bus $i$ and bus $j$ and $P_{i j}^{\min } / P_{i j}^{\max }$ are the minimum and maximum power capacity limit of the transmission line between the two buses respectively.

\subsection{Uncertainty modeling of solar PV}

Solar energy is affected by different parameters such as temperature and solar irradiance. The main parameter which has the largest effect on the PV unit is the solar irradiance. The PV unit output power, $P_{t}^{p v}$, can be expressed as [22]:

\footnotetext{
On the dispatch of minigrids with large penetration levels of variable renewable energy (Anan A. Dweekat)
} 


$$
P_{t}^{p v}=\sum_{M} A_{p v} \times \eta_{p v} \times i r r_{t}
$$

where $M$ is the number of PV units, $A_{p v}$ is the area of the PV unit, $\eta_{p v}$ is the PV unit efficiency and $i r r_{t}$ is the solar irradiance at time $t$. The solar irradiance is modeled as a stochastic random variable, which is fitted into a probability density function (PDF) as in $[22,23]$. Beta distribution is the PDF used, since it was found to give the best fit of the solar irradiance data.

\subsection{Power generation and load balance constraint}

The summation of the output power generated by the conventional DGs in the minigrid should be greater than or equal to the load after subtracting the output power of the PV DGs. This can be modeled as:

$$
\sum_{i} P_{i, t} \geq L_{t}-P_{t}^{p v}
$$

where $L_{t}$ is the load demand at the minigrid. The output power of the PV is considered as a negative load. The PV generation is disbursed in the minigrid as small DGs close to the end users [24]. The above equations form the minigrid stochastic optimization dispatch approach and the MILP is used to solve it. The dispatch approach and its solution are implemented using general algebraic modeling system (GAMS) software [25], on the modified IEEE 14-bus test system as discussed in the next section.

\section{RESULTS AND DISCUSSION}

The IEEE 14-bus system, representing a minigrid, consist of 20 transmission lines and 3 conventional generating units as shown in Figure 1. These conventional generators are connected at busses 1 , 2 and 3 respectively. Data for the generating units were taken from [26], whereas the system line data were taken from [17].

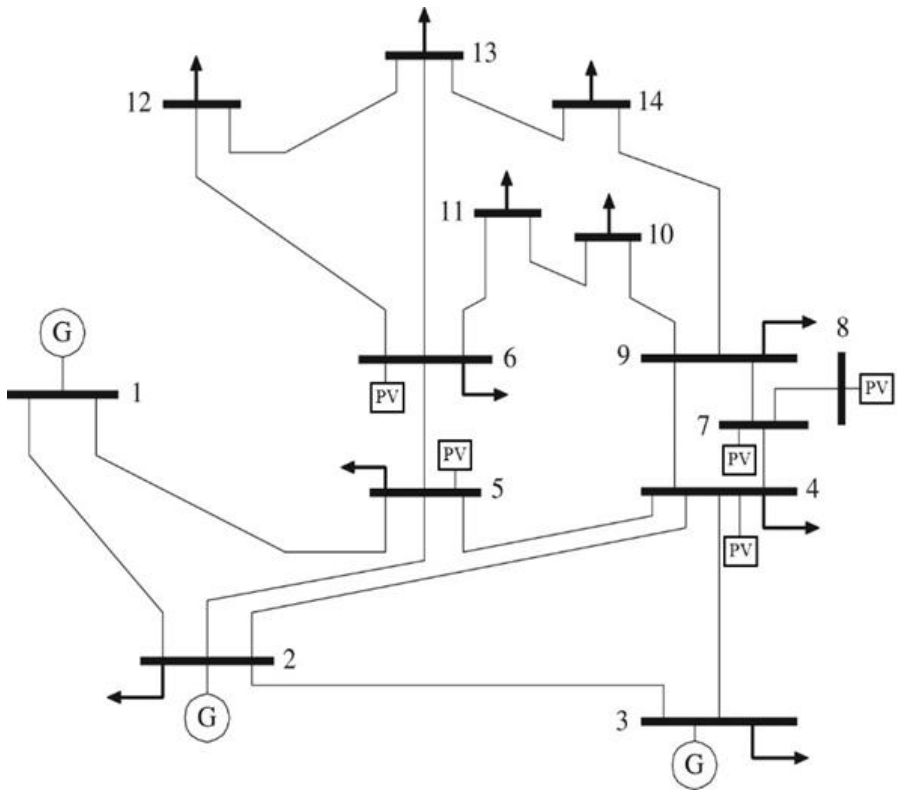

Figure 1. The modified IEEE 14-bus test system

\subsection{Minigrid generation scheduling without solar PV}

In this first case, the proposed model is applied to the IEEE 14-bus system without considering solar PV generation. The equations considered (1)-(16) and (18). In other words, $P_{t}^{p v}$ equals zero in (18). The total load demand and output power of each conventional generating unit are shown in Figure 2. The first unit is committed almost invariably over the entire dispatch horizon, indicating that operation cost of unit \#1 is the lowest among the three units. The peak load is $259 \mathrm{MW}$. When the load starts picking up after 6 am, the second unit will kick in increasing its output gradually to satisfy the load demand on its highest peaks. The total operation cost considering transmission lines limits is $27,769.7662 \mathrm{USD} /$ day, for this case. 


\subsection{Minigrid generation scheduling considering solar PV}

In this case, five solar PV units are disbursed at buses 4, 5, 6, 7 and 8, as shown in Figure 1. The load curve is maintained as shown in Figure 2.

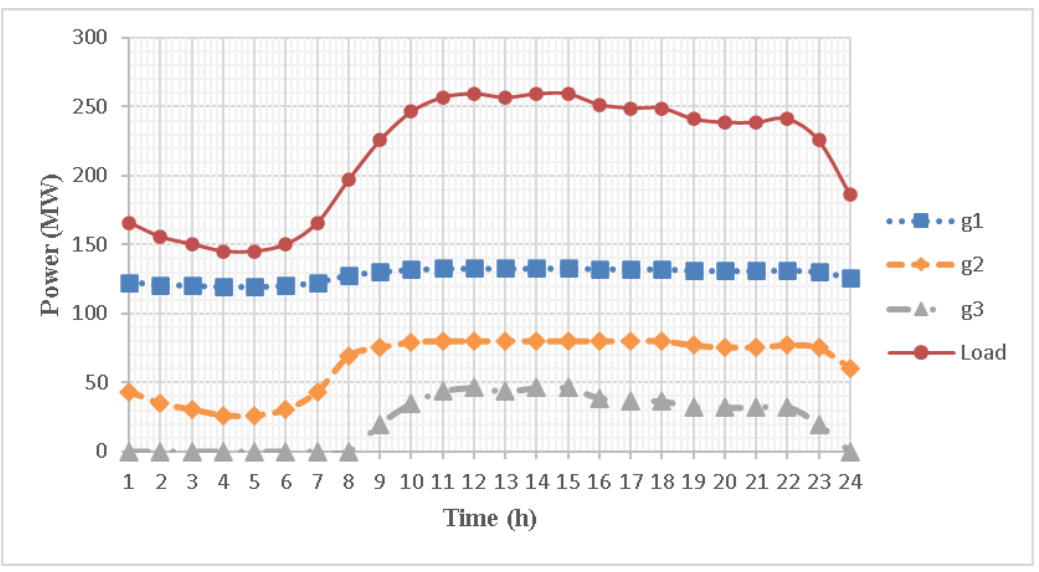

Figure 2. Hourly generation dispatch for the modified IEEE 14-bus system with no solar PV

The solar irradiance data of the city of Austin, Texas, USA is considered in this paper. The data were taken from an online source of the University of Texas Solar Energy Laboratory from the National Renewable Energy Lab (NREL). The city is located at Latitude: $30.29^{\circ} \mathrm{N}$ and Longitude: $97.74^{\circ} \mathrm{E}$ [18]. The data are then fitted into a Beta PDF, as mentioned in an earlier section, which was found to provide the best fit of the given data. The solar irradiance data is thus modeled as a stochastic random variable and included in (17) to capture the effect of uncertainty in PV output power. As shown in (1)-(19) are included in the minigrid dispatch model. The developed dispatch model is applied on the modified IEEE 14 bus system at two different PV penetration levels.

\subsubsection{0\% solar PV penetration level-stochastic modeling}

The penetration level of the PV units is $20 \%$ of the peak load. Figure 3 shows the solar PV output power and the output power of each conventional generating unit in the 14-bus system. Apparently conventional generation patterns are affected by the variability nature of the solar irradiance as shown in Figure 3. When the load is increasing after $6 \mathrm{am}$, the PV output power is also increasing, which triggers the second unit to ramp up to satisfy the net load, while the third unit remains off. The power generated by unit 2 and 3 have decreased as compared with the previous case with no solar PV. The effect of solar energy variability in this case remains small, though, due to the low penetration level. The total operation cost of the minigrid dispatch with a 20\% solar PV penetration level is found to be 26,029.1309 USD/day. It is clear that the operation cost has decreased as compared with the case of no solar PV. This is solely attributed to the zero-operation cost characterizing the dispatch of solar PV among the generation mix.

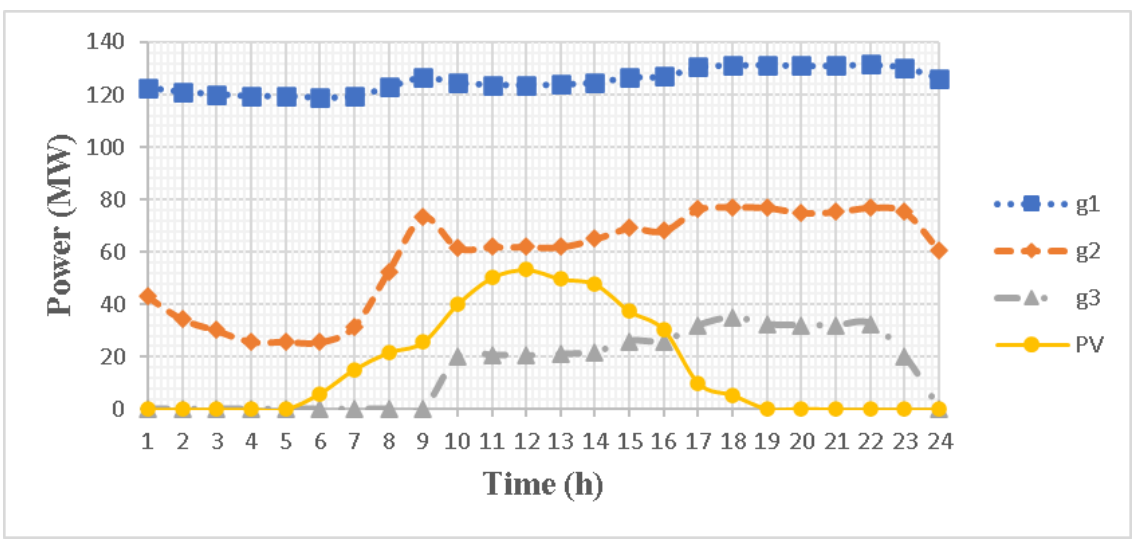

Figure 3. Dispatch of the modified IEEE 14-bus system with 20\% PV penetration level

On the dispatch of minigrids with large penetration levels of variable renewable energy (Anan A. Dweekat) 


\subsubsection{0\% PV penetration level-deterministic modeling}

At a $20 \%$ PV penetration level of the peak load, the capacity credit of solar PV is considered as 46.04\% [27]. In contrast with stochastic PV modeling carried out in the previous test case using PDF, the capacity credit of solar PV is a deterministic quantification of the variable PV output. In this model, the solar PV output power remains constant, at partial value, throughout the sunlight hours in the dispatch period. Figure 4 shows the dispatch results. The total operation cost in this case is 25,380.8725 USD/day, which is lower than the operation cost obtained in case A. This is due to the fact that the amount of energy produced by the solar PV in the capacity credit deterministic model is higher than its counterpart in the stochastic model using PDF. This confirms that the capacity credit deterministic model gives optimistic results that are not usually conforming with practical case studies of solar PV output.

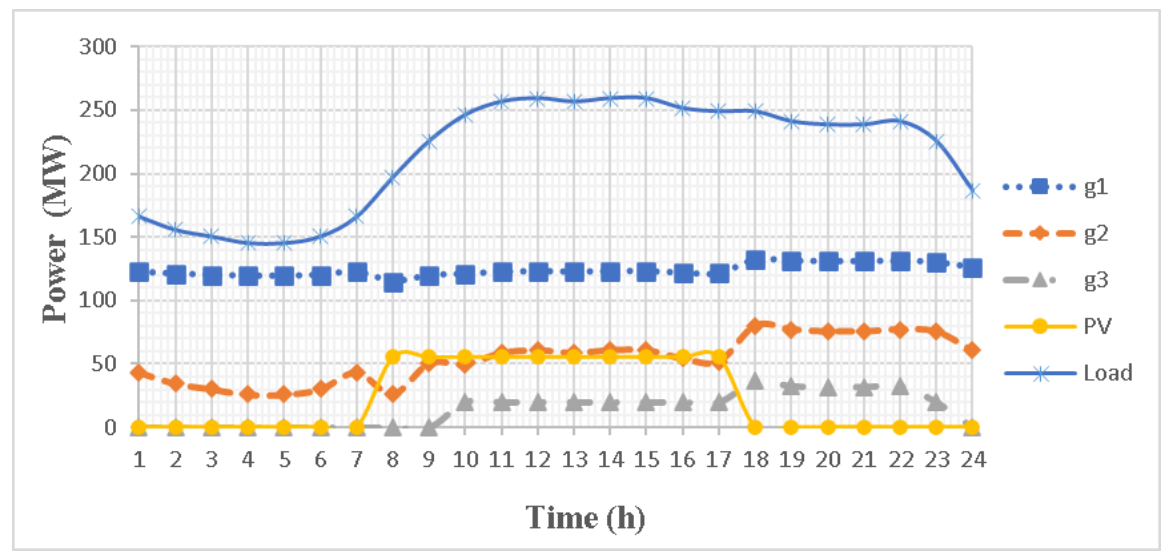

Figure 4. Modified IEEE 14-bus system dispatch at 20\% PV penetration level using a deterministic model

\subsubsection{5\% solar PV penetration level level-stochastic modeling}

The solar PV penetration level is increased to $55 \%$ in this case. The output power of units g1, g2, and g3 have decreased as shown in Figure 5, when the solar PV output power kicks in gradually between t6 and $\mathrm{t} 18$. Solar PV has delayed the commitment of $\mathrm{g} 3$ by 7 hours to t16, as shown in Figure 5, instead of $\mathrm{t} 9$ in Figure 2 and Figure 3. Following the load curve, as the solar energy starts the downward trend in its parabolic generation curve at $\mathrm{t} 14, \mathrm{~g} 2$ gradually ramps up to make up for the generation shortfall. When the PV output drops further at t16, g3 is called upon to ramp up its energy output. Eventually, the combined output of the three conventional generators along with solar PV generation will meet the load demand of the minigrid. This result highlights the efficacy of the proposed approach in capturing the intermittent characteristics of solar PV generation with a reasonable accuracy as well as maintaining an economic dispatch of the minigrid generation resources. The total operation cost has diminished to 22,697.0290 USD/Day, which is the lowest compared to previous cases.

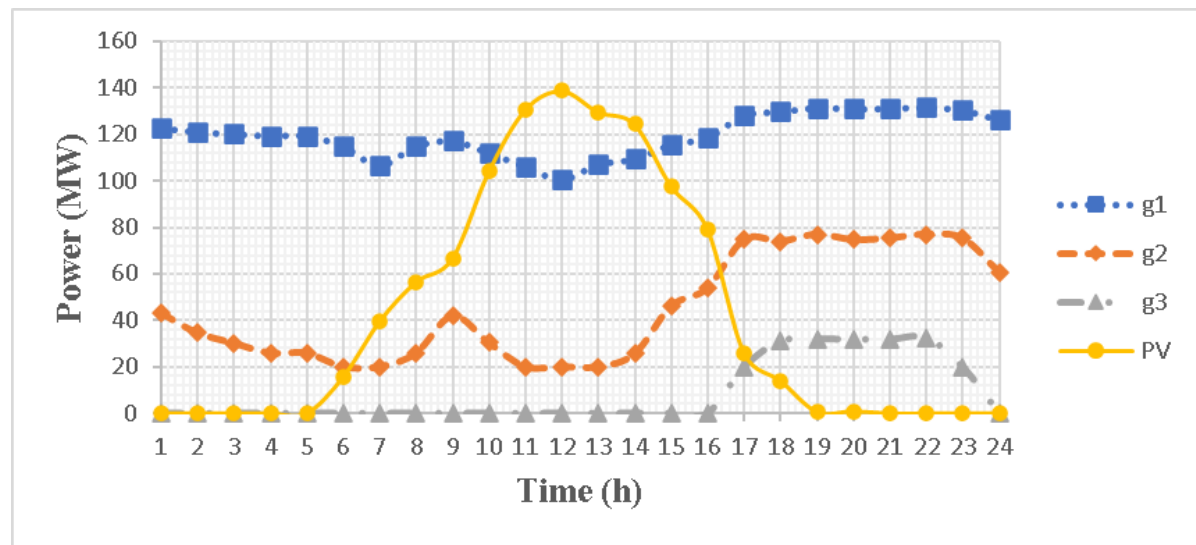

Figure 5. Stochastic scheduling of the modified IEEE 14-bus system at 55\% PV penetration level 
Since solar PV power is modeled as a negative load, the net load demand is the difference between the minigrid total end consumer load and the power generated from the PV units. The combined output of the three thermal conventional generators g1, g2, and g3 as well as PV output power and total minigrid load are shown in Figure 6. It is clear that the proposed minigrid scheduling approach managed to reduce thermal generation output to give room for clean and free RE coming from solar PV units. As solar energy subsides after sunsets and at night, thermal generation ramps up/down accordingly to match the load in every hour of the 24-h dispatch period. Table 1 summarizes the total operation cost corresponding to various PV penetration levels.

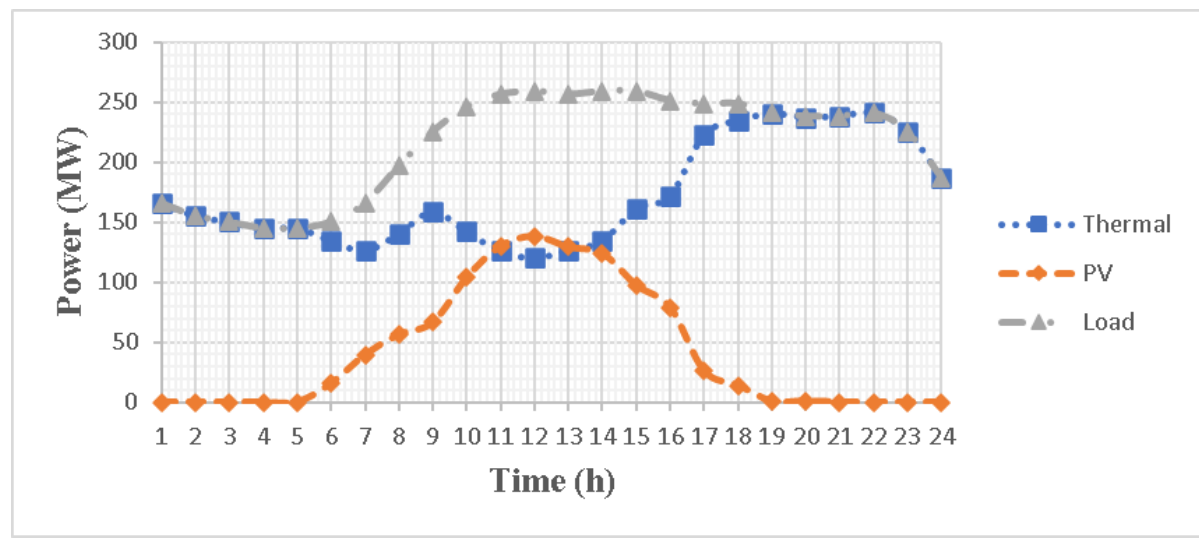

Figure 6. Load demand, combined thermal generation output, and solar PV output at 55\% penetration level

Table 1. Total operation cost at different PV penetration levels

\begin{tabular}{cc}
\hline Operation cost & PV penetration level \\
\hline $27,769.7662$ USD/day & $0 \%$ \\
$26,029.1309$ USD/day & $20 \%$ \\
$22,697.0290$ USD/day & $55 \%$ \\
\hline
\end{tabular}

\subsection{One-week stochastic minigrid dispatch}

In this case the proposed approach is applied on the modified IEEE 14-bus testbed system for one week, at a $45 \%$ PV penetration level. Figure 7 shows the generation schedule over 168-hour (one week). It is evident that the thermal generators follow the load closely. Yet, when solar PV energy becomes available, thermal generators are scaled back to allow for the full exploitation of the clean RE. The total operation cost of one-week dispatch is $169,049.0092$ USD/week. Nonetheless, the total operation cost without considering solar energy at all amounts to 194,506.1208 USD/week. This means there is a net saving of 25,457.11 USD weekly, when solar PV generation is included in the minigrid generation mix.

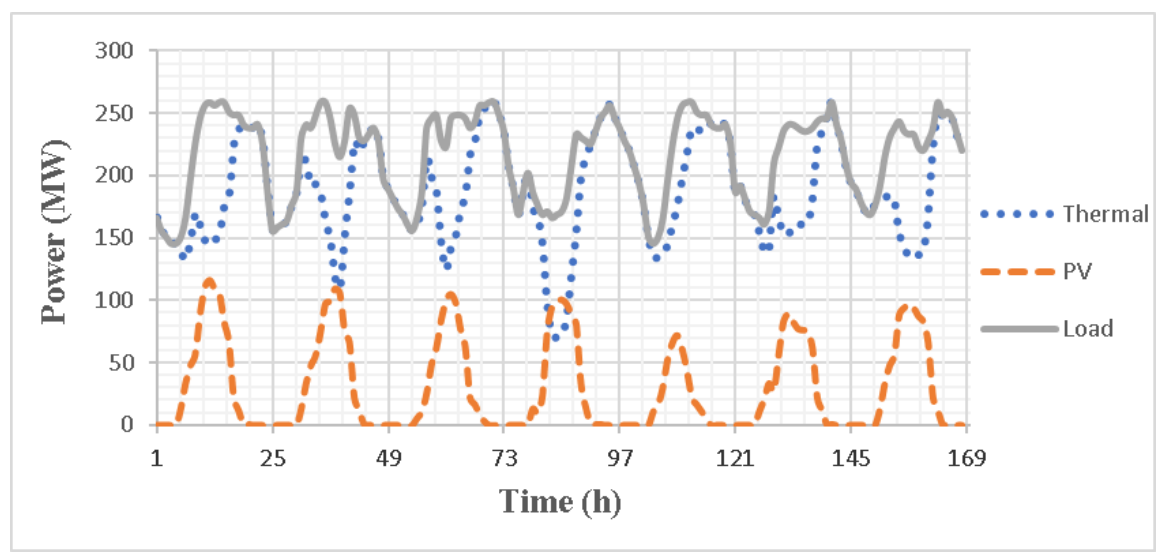

Figure 7. A one-week dispatch of the modified IEEE 14-bus system at $45 \%$ PV penetration level 


\section{CONCLUSION}

Hourly generation dispatch of a minigrid that has DGs, including renewable solar PV generation, serving minigrid's end consumer load is presented in this paper. Solar irradiance is the parameter that has the dominant effect on PV power output and is highly sensitive to the illuminance of the sun in daylight conditions. Solar irradiance was therefore represented as a stochastic random variable that was statistically fitted into a Beta PDF using actual data. The minigrid dispatch problem is formulated as a stochastic nonlinear optimization problem to minimize the operation costs. The problem is then linearized to fit into an MILP structure, which has robust solution techniques. The proposed approach is applied to a modified IEEE 14-bus test system and many test scenarios were conducted. Results show that as the solar PV penetration level increases, the operation costs decrease. Also, the stochastic modeling of solar PV is more reflective of PV intermittency as compared with capacity credit-based deterministic modeling. The adequacy of the proposed approach was further validated on a 168-hour, one week minigrid scheduling. Integration of multiple variable RES with disparate characteristics in a minigrid and the coordination between them is currently under investigation.

\section{ACKNOWLEDGEMENTS}

This paper is supported by the Malaysian Ministry of Education (MOE), under the Fundamental Research Grant Scheme (FRGS) and Universiti Malaysia Sarawak (UNIMAS), Grant No: F02/FRGS/1620/2017.

\section{REFERENCES}

[1] K. Rahbar, C. C. Chai, and R. Zhang, "Energy Cooperation Optimization in Microgrids with Renewable Energy Integration," IEEE Transactions on Smart Grid, vol. 9, no. 2, pp. 1482-1493, 2018.

[2] J. Jurasz, F. A. Canales, A. Kies, M. Guezgouz, and A. Beluco, "A Review on the Complementarity of Renewable Energy Sources: Concept, Metrics, Application and Future Research Directions,” Solar Energy, vol. 195, pp. 703-724, 2020.

[3] X. Xu et al., "Maximum Loadability of Islanded Microgrids with Renewable Energy Generation," IEEE Transactions on Smart Grid, vol. 10, no. 5, p. 4696-4705, 2019.

[4] S. R. Sinsel, R. L. Riemke, and V. H. Hoffmann, "Challenges and Solution Technologies for the Integration of Variable Renewable Energy Sources-A Review,” Renewable Energy, vol. 145, pp. 2271-2285, 2020.

[5] P. A. Ostergaard, N. Duic, Y. Noorollahi, H. Mikulcic, and S. Kalogirou, "Sustainable Development Using Renewable Energy Technology," Renewable Energy, vol. 146, pp. 2430-2437, 2020.

[6] J. O. Petinrin and M. Shaaban, "Renewable Energy for Continuous Energy Sustainability in Malaysia," Renewable and Sustainable Energy Reviews, vol. 50, pp. 967-981, 2015.

[7] A. Zakaria, F. B. Ismail, M. S. H. Lipu, and M. A. Hannan, "Uncertainty Models for Stochastic Optimization in Renewable Energy Applications,” Renewable Energy, vol. 145, pp. 1543-1571, 2020.

[8] T. A. O. Li, W. Hu, S. Member, X. Xu, and S. Member, "Optimized Operation of Hybrid System Integrated with MHP, PV and PHS Considering Generation/Load Similarity," IEEE Access, vol. 7, pp. 107793-107804, 2019.

[9] S. R. Salkuti, "Optimal Operation Management of Grid-Connected Microgrids Under Uncertainty," Indonesian Journal of Electrical Engineering and Computer Science, vol. 16, no. 3, pp. 1163-1170, 2019.

[10] K. H. D. Tang, "Hydroelectric Dams and Power Demand in Malaysia: A Planning Perspective," Journal of Cleaner Production, vol. 252, pp. 119-127, 2020.

[11] E. D. F. Moscardini Júnior and R. Rüther, "The Influence of the Solar Radiation Database and the Photovoltaic Simulator on the Sizing and Economics of Photovoltaic-Diesel Generators," Energy Conversion and Management, vol. 210, pp. 112-121, 2020.

[12] A. Sharma, S. Agrawal, and J. Urpelainen, "The Adoption and Use of Solar Mini-Grids in Grid-Electrified Indian Villages,” Energy for Sustainable Development, vol. 55, pp. 139-150, 2020.

[13] T. Van Gevelt, C. Canales Holzeis, F. George, and T. Zaman, "Indigenous Community Preferences for Electricity Services: Evidence from a Choice Experiment in Sarawak, Malaysia," Energy Policy, vol. 108, pp. 102-110, 2017.

[14] A. A. Dweekat and M. Shaaban, "Optimal Microgrid Energy Scheduling with Variable Solar PV Generation," 2019 International UNIMAS STEM 12th Engineering Conference, pp. 22-27, 2019.

[15] Y. K. Wu, G. T. Ye, and M. Shaaban, "Analysis of Impact of Integration of Large PV Generation Capacity and Optimization of PV Capacity: Case Studies in Taiwan," IEEE Transactions on Industry Applications, vol. 52, no. 6, pp. 4535-4548, 2016.

[16] S. R. Salkuti, "Solving Optimal Generation Scheduling Problem of Microgrid Using Teaching Learning Based Optimization Algorithm," Indonesian Journal of Electrical Engineering and Computer Science, vol. 17, no. 3, pp. 1632-1638, 2020.

[17] A. J. Wood, B. F. Wollenberg, and G. B. Sheblé, "Power Generation, Operation, and Control, 3rd ed. NJ," USA: John Wiely \& Sons: Hobbkin, 2014.

[18] W. S. Tan, M. Shaaban, and M. Z. A. A. Kadir, "Stochastic Generation Scheduling with Variable Renewable Generation: Methods, Applications, and Future Trends," IET Generation, Transmission and Distribution, vol. 13, no. 9 , pp. 1467-1480, 2019. 
[19] S. A. S. Lafta, A. H. Ali, M. M. Kareem, Y. A. Hussein, and A. H. Ali, "Robust Operation of Microgrid Energy System Under Uncertainties and Demand Response Program," Indonesian Journal of Electrical Engineering and Computer Science, vol. 17, no. 2, pp. 1005-1013, 2019.

[20] W. Tan and M. Shaaban, "A Hybrid Stochastic/Deterministic Unit Commitment Based on Projected Disjunctive MILP Reformulation," IEEE Transactions on Power Systems, vol. 31, no. 5, pp. 586-588, 2016.

[21] M. Carrión and J. M. Arroyo, "A Computationally Efficient Mixed-Integer Linear Formulation for the Thermal Unit Commitment Problem,” IEEE Transactions on Power Systems, vol. 21, no. 3, pp. 1371-1378, 2006.

[22] G. Mokryani, A. Majumdar, and B. C. Pal, "Probabilistic Method for the Operation of Three-Phase Unbalanced Active Distribution Networks," IET Renewable Power Generation, vol. 10, no. 7, pp. 944-954, 2016.

[23] V. K. Jadoun, V. C. Pandey, N. Gupta, and K. R. Niazi, "Integration of Renewable Energy Sources in Dynamic Economic Load Dispatch Problem Using an Improved Fireworks Algorithm," IET Renewable Power Generation, vol. 12, no. 9, pp. 1004-1011, 2018.

[24] A. Samadi, L. Soder, E. Shayesteh, and R. Eriksson, "Static Equivalent of Distribution Grids with High Penetration of PV Systems,” IEEE Transactions on Smart Grid, vol. 6, no. 4, pp. 1763-1774, 2015.

[25] R. E. Rosenthal, "GAMS - A User's Guide," GAMS Development Corporation, vol. 1, no. 3, p. 22, 2007. [Online]. Available: https://www.un.org/en/development/desa/policy/mdg_workshops/training_material/gams_users_guide.p df. [Accessed June 30, 2020].

[26] J. Nanda, L. Hari, and M. L. Kothari, "Economic Emission Load Dispatch with Line Flow Constraints Using a Classical Technique," IEE Proceedings-Generation, Transmission and Distribution, vol. 141, no. 1, pp. 1-10, 1994.

[27] M. Ding and Z. Xu, "Empirical Model for Capacity Credit Evaluation of Utility-Scale PV Plant," IEEE Transactions on Sustainable Energy, vol. 8, no. 1, pp. 94-103, 2017. 